\title{
Resveratrol Treatment Protects Tendons from Obesity and Insulin Resistance Effects
}

\author{
T. M. Da Ré Silva', S. H. Ferreira da Cruz', D. Majolli Andre², P. Pires Marques³, \\ L. Prado de Oliveira ${ }^{4}$, C. Pedrozo Vieiraa ${ }^{5}$ F. Da Ré Guerra ${ }^{1}$ \\ 1 Department of Anatomy, Institute of Biomedical Science, Federal University of Alfenas - UNIFAL-MG, Alfenas \\ (MG), Brazil \\ 2 Department of Pharmacology, Faculty of Medical Sciences, State University of Campinas, Cidade Universitária \\ Zeferino Vaz - Barão Geraldo, Campinas (SP), Brazil \\ 3 Department of Medicine, José do Rosário Vellano University-Unifenas, Alfenas (MG), Brazil \\ ${ }^{4}$ Department of Cell Biology, Institute of Biology, State University of Campinas, Cidade Universitária Zeferino \\ Vaz - Barão Geraldo, Campinas (SP), Brazil \\ ${ }^{5}$ Department of Ophthalmology, The University of Alabama at Birmingham, Birmingham (AL), U.S.A.
}

\section{CORRESPONDING AUTHOR:}

Flávia Da Ré Guerra

Department of Anatomy

Institute of Biomedical Science

Federal University of Alfenas -

\section{UNIFAL-MG}

Gabriel Monteiro da Silva St 700 Alfenas (MG) 37130-001, Brazil

E-mail: unifal.flavia@gmail.com

DOI:

10.32098/mltj.04.2021.03

LEVEL OF EVIDENCE: 2B

\begin{abstract}
SUMMARY
Background. Obesity and Insulin resistance (IR) are conditions that cause metabolic disorders and secondary effects, including musculoskeletal complications making tendon prone to lesions. Antidiabetic drugs presents several side effects, making fundamental the pursue of alternative treatments, to improve patient's quality of life. Resveratrol (RSV) can re-establish insulin sensitivity and regulate glycemic levels. It also presents a range of other biological properties such as: antioxidant, anti-inflammatory, neuroprotective, anti-aging, anti-cancer, prevention of cardiovascular disease, and improvement in mitochondrial function. The study evaluates RSV's effects in the calcaneal tendon in insulin-resistant obese animals. Methods. 40 male mice (C5B16) were distributed in 8 groups: Non-obese Control; Non-obese Insulin-treated; Non-obese RSV-treated; Non-obese Insulin and RSV-treated; Obese; Obese insulin-treated; Obese RSV-treated; Obese Insulin and RSV-treated. IR was induced by a hyperlipidemic AIN-93 diet and attested through an insulin tolerance test. RSV was administered daily via gavage $(100 \mathrm{mg} / \mathrm{kg})$ for two weeks, starting on the $10^{\text {th }}$ week of life until euthanasia, when tendons were removed for testing. Results. Resveratrol treatment demonstrated a protective effect over tendons in obese, insulin-resistant animals, reducing the activity of different MMPs, MMP-2, and MMP-9, and avoiding protein content reduction, maintaining its levels similar to healthy animals. Conclusions. Resveratrol treatment reduces the activity of, MMP-2 and MMP-9, and also avoids protein content degradation. Its effects over inflammation could be helpful addressing IR patients, helping not only to control the disease, but also making the tendon lesion treatment more efficient.
\end{abstract}

\section{KEY WORDS}

Diabetes; extracellular matrix; insulin resistance; metalloproteinases; non-collagenous protein.

\section{BACKGROUND}

Obesity and insulin resistance (IR) impairs the ability of muscle cells to uptake and store glucose and triglycerides, which generates a high level of glucose and triglycerides in the bloodstream (1). IR leads to decreased sensitivity and responsiveness to insulin, therefore, it plays a significant pathophysiologic role in DM2. DM2 is a metabolic dysfunction, in which the insulin function is impaired, 
whether because of the IR or the pancreas beta cells are not producing enough insulin. This chronic disease results in systemic inflammation and high blood glucose levels: hyperglycemia (2). IR is usually present in older adults but has become prevalent at all ages on individuals who are overweight and sedentary, which are also the chief risk factors of DM2. Several studies have demonstrated the association between these diseases and musculoskeletal disorders (2-5).

It is believed that IR impairs inflammatory response and alters different connective tissues constitution, compromising its functions, and damaging structures like tendons (4). Furthermore, hyperglycemia contributes to the formation of advanced glycation end-products (AGE), which modifies tendon physical properties (5). AGEs and oxidative stress mediators alter the organization of the tendons ECM and modifies its thermal stability by directly inducing collagen crosslinks. In addition, the effects of AGEs on cells promote an increase in the activity of transaglutaminase enzymes, contributing to the formation of calcification in diabetic tendons (6).

According to Bedi et al., the risk of tendon lesion recurrence is higher in diabetic patients without glycemic control, showing that proper blood glucose levels can preserve the biologic traits of the tendons and reduce the risks of infection and other complications following tissue repair (4). In that matter, another study showed that muscle work during aquatic excercises improves the consumption of corporal glucose, therby reducing blood glucose levels. Nonetheless, physical exercise was not enough to solve the imbalance that leads to alteration of the tendons structural homogeneity and its biomechanical properties (7).

The calcaneal tendon is one of the most susceptible to lesions and the most prone to spontaneous rupture. Several factors such as age, gender, obesity, insulin resistance, and diabetes can increase the odds of lesion incurrence (8). Animals with IR present impairment in tendon mechanical properties and collagen bundles disorganization (6). The repair process of such structures is characterized by accretion of cicatricial tissue with increased cellularity. These factors affect the tendon attributes, necessary to fulfill its functions (2). Usual treatments against diabetes and IR are based on drugs, but weight gain and hypoglycemia are recurrent side effects. To avoid these complications, several alternative therapies are currently under investigation, including phytotherapy (9). Recent studies demonstrated that resveratrol (RSV), a polyphenolic compound, may be employed in IR treatment due to its properties (9).

RSV is a natural stilbene polyphenol found especially in grapes and red wine. It presents anti-inflammatory, anti-diabetic, and anti-tumoral properties, also able to help against neurodegenerative diseases (10). This substance may have the capacity to assist in the repair of tendon lesions caused by IR, since it might aid in diabetes and IR treatment - risk factors that impairs tendon recovery - and also act against the inflammatory process.

Tendon repair and mechanical properties are altered in IR and patients also present articular limitations due to tendon stiffness. It is common for IR patients to develop foot ulcers, resulting from pathological changes in nerves and tendons. The etiology of this ulcer is a combination of neurological degeneration and changes in the tendon structure. Although not fatal, it is a debilitating condition that may result in amputation as a form of treatment. Thus, the prevention or even the efficient treatment of tendon injuries could be the best way to prevent the occurrence of ulcers (11). The longevity of these patients has been increasing, but also has the incidence of tendon related problems, reflecting the urgency of new therapies (12).

\section{MATERIALS AND METHODS}

Animal care was in accordance with the Basic principles and recommendations in clinical and field Science Research (13) and is consistent with the ethical principles of animal experimentation adopted by the Brazilian College of Animal Experimentation (COBEA). The present study was approved by the Committee on Ethics in Animal Experiment (CEEA) of the State University of Campinas UNICAMP (Protocol n ${ }^{\circ}$ 3502-1).

\section{Animals and experimental groups}

Male 4-week-old mice from the C57BL/6 line were used. The animals were obtained from the Multidisciplinary Center for Biological Research (CEMIB) at UNICAMP and kept at $24{ }^{\circ} \mathrm{C}$, with a daily lighting period of $12 \mathrm{~h}$, with water and food ad libitum in the vivarium of the Pharmacology Department of FCM/UNICAMP. A total of 40 young adult male mice (C57B16) were distributed in 8 groups:

- non-obese control group (untreated);

- non-obese Insulin-treated group;

- non-obese Resveratrol (RSV) treated group;

- non-obese Insulin + RSV group;

- obese group;

- obese Insulin-treated group;

- obese Resveratrol (RSV) -treated group;

- obese Insulin + RSV group.

After the appropriate treatments, the animals suffered euthanasia through the deepening of anesthesia with isoflurane followed by cervical dislocation. The calcaneal tendons were removed for further analysis. 


\section{Inducing obesity with hyperlipidic diet}

Obesity was induced by replacing the standard mice feed (AIN-93) with a high-fat diet of the Pragsoluções brand, for 12 weeks. The lean groups received a standard commercial diet (10\% lipid, $70 \%$ carbohydrate and $20 \%$ protein) from NUVILAB (14). Both diets and water were offered ad libitum.

\section{Resveratrol treatment}

Obese and lean mice were treated daily with resveratrol (100 $\mathrm{mg} / \mathrm{kg}$ ) per gavage, for two weeks (15). The treatment started concomitantly with the first immunization $\left(10^{\text {th }}\right.$ week), ending after the last sensitization and challenge with ovalbumin $\left(12^{\text {th }}\right.$ week).

\section{Insulin tolerance test (ITT)}

The animals were kept at fast for 6 hours and their blood was collected through the caudal vein. The blood glucose level was assessed using a glucometer (Accu-Chek Performa, Roche Diagnostics).

Animals received, after fasting, an intraperitoneal injection of insulin $(1 \mathrm{IU} / \mathrm{kg})$. Blood was collected from the tail at 0 , 5, 10, 15, 20, 25 and $30 \mathrm{~min}$, to measure glucose levels. The rate of glucose decay was then calculated using the linear regression curve of the Neperian logarithm of glucose versus time. This value was assumed as the glucose decay constant after ITT and expressed as a percentage per minute (16).

\section{Insulin administration and euthanasia}

Animals were anesthetized with ketamine $(100 \mathrm{mg} / \mathrm{kg})$ and xylazine $(10 \mathrm{mg} / \mathrm{kg})$. Then the abdominal cavity was opened and the animals received an intravenous injection of insulin (Humulin regular; 1 UI per animal in the inferior vena cava). After 5 minutes, the animals were euthanized by cervical displacement and the calcaneal tendons removed for analysis.

\section{Extraction procedures}

Tendons were cut into small pieces and homogenized with extraction buffer (Tris-HCl $50 \mathrm{mM}, \mathrm{pH} 7.4, \mathrm{NaCl} 0.2 \mathrm{M}$, Triton X-100 0.1\%, $\mathrm{CaCl}_{2} 10 \mathrm{mM}$ and protease inhibitor $10 \mu \mathrm{l} / \mathrm{ml}$ ). For every $30 \mathrm{mg}$ of tissue, $100 \mu \mathrm{l}$ of buffer was used. Proteins' extraction took place in ice, under stirring, for 2 hours, after which the material was centrifuged for 20 minutes at $4000 \mathrm{rpm}$, under refrigeration. After supernatant removal, the precipitate was resuspended with $1 / 3$ of the initial volume, heated for 5 minutes at $60^{\circ}$, and centrifuged again. The supernatants of both steps were mixed (17).

\section{Non-collagenous proteins quantification}

Non-collagenous proteins (NCP) were quantified following the Bradford method (18) using Coomassie Brilliant Blue G-250. Bovine serum albumin (BSA) was used as standard and readings were performed using an HP 8452 A Diode Array spectrophotometer at $595 \mathrm{~nm}$.

\section{Zymography}

For the electrophoresis following the extraction, researchers used a $10 \%$ polyacrylamide gel containing $2 \mathrm{mg} / \mathrm{ml}$ gelatin and applied $20 \mu \mathrm{g}$ of protein (15). After the run, gels were placed in incubation buffer for $24 \mathrm{~h}$ (Tris- $\mathrm{HCl} 50 \mathrm{mM}$ $\mathrm{pH} 8.4, \mathrm{CaCl}_{2} 5 \mathrm{mM}$, and $\mathrm{ZnCl}_{2} 1 \mu \mathrm{M}$ ). To visualize the bands, the gels were stained with Coomassie Brilliant Blue R-250 and destained using methanol $30 \%$ and acetic acid $10 \%$ solution in water.

The gels obtained were imaged and band densitometry was measured in pixels using Li-Cor Image Studio Lite 5.2.5.

\section{Biomechanical analysis}

Tendon biomechanics were analyzed using a texturometer TA.XT plus, from Stable Micro Systems. Five tendons from each experimental group were measured in their three dimensions using a digital caliper and the transversal section of the tendon $(\mathrm{A})$ was calculated.

During the test, both the ends of the tendons are fixed in the machine, which applies an increasing load at a constant rate of $20 \mathrm{~mm} / \mathrm{min}$ until tendon rupture. The force necessary to rupture the tendon was designated as failure load (F). With this parameter and the tendon displacement, it was possible to calculate the Stress in $\mathrm{MPa}(\sigma=\mathrm{F} / \mathrm{A})$; the strain in $\%(\varepsilon=$ displacement/initial length), and the Young Modulus $(E=\sigma / \varepsilon)$.

\section{Statistical analysis}

All results are here expressed as mean \pm standard deviation. Data from different experimental groups were analyzed by analysis of variance (oneway ANOVA) followed by the Tukey test. The level of significance was $\mathrm{p}<0.05$. The analysis were carried out in GraphPad Prism ${ }^{\circledR}$ (GraphPad Software, La Jolla, CA, USA).

\section{RESULTS}

Data obtained through zymography can be observed in figure 1. The zymogram revealed the presence of metalloproteinase-2 and -9 (MMP-2 and MMP-9). The densitometries are plot in figure 2. It is possible to observe that obese 


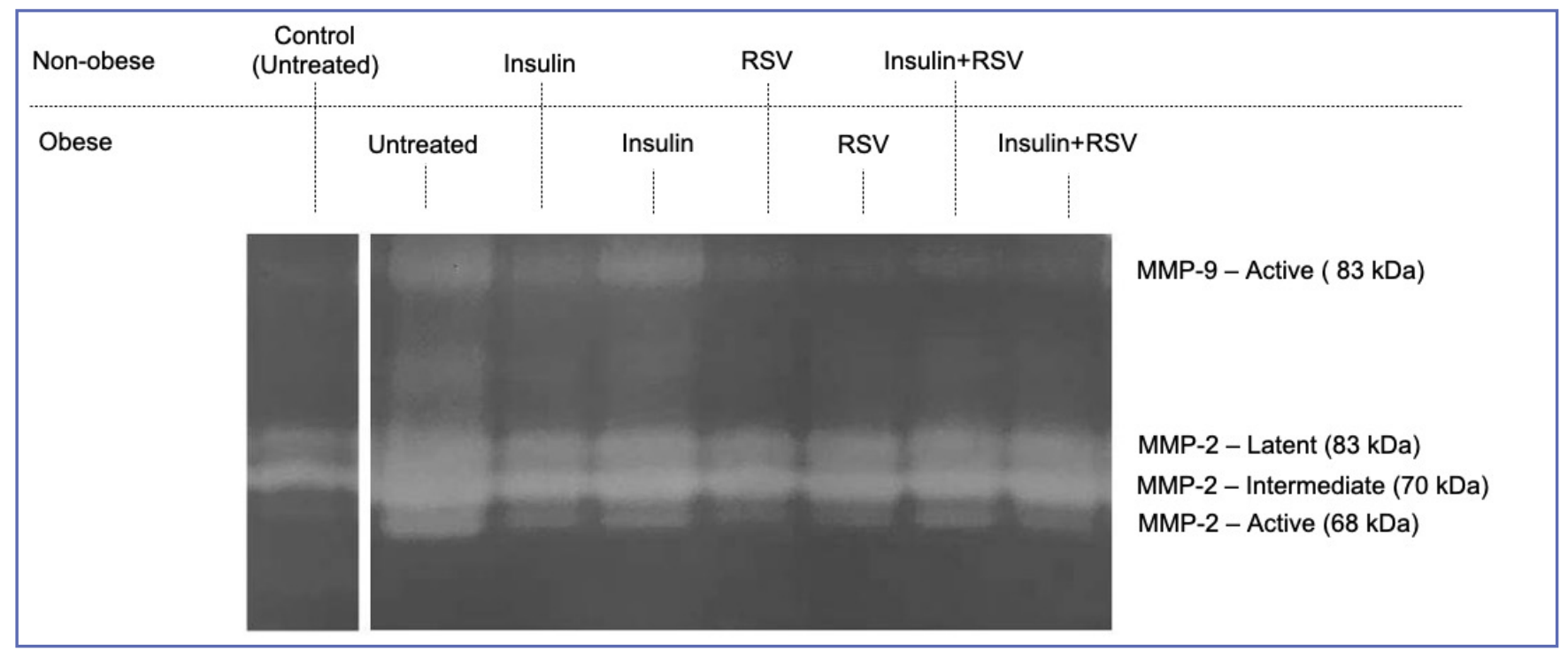

Figure 1. Zymography of the different groups revealing the presence of different MMPs.

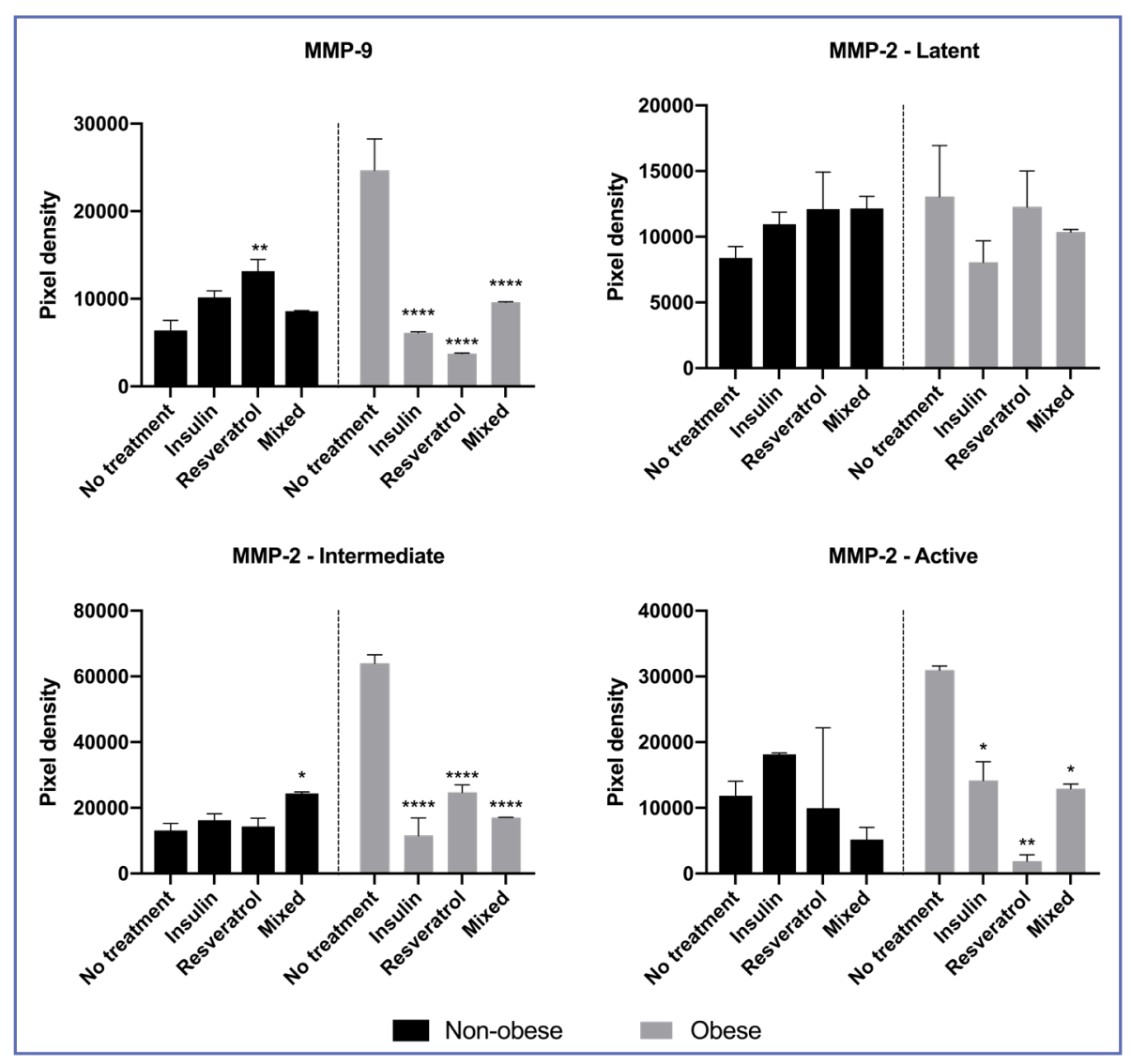

Figure 2. Band densitometry obtained from zymography of MMPs - 2 and - 9.

The * means the treatment caused a significative difference compared to the untreated group inside the same set (obese/non-obese). *p $<0.05 ;{ }^{* *} \mathrm{p}<0.01 ;{ }^{* * *} \mathrm{p}<0.001 ;{ }^{* * * *} \mathrm{p}<0.0001$. animals, without any kind of treatment, presented the highest amount of most MMPs found. Insulin and RSV were both able to reduce the concentration of MMP-9 and the intermediate and active isoforms of MMP-2. Resveratrol was particularly able to reduce the concentration of the active MMP-2.

In figure 3 we can see that obesity by itself caused a reduction in the protein content compared to the control group. Interestingly, any treatment containing insulin (even on non-obese animals) caused a reduction in the protein content compared to the control group (figure 3). Nonetheless, animals receiving only RSV (both obese and otherwise) presented no reduction in the protein content.

The biomechanical results are plotted in figure 4. As it can be observed, the strength necessary to cause tendon rupture (Stress) was much lower in obese animals, but insulin was able to counter the obesity effect. The Young Modulus followed the same pattern as the Stress. No difference was observed in any group regarding tendon Strain. 


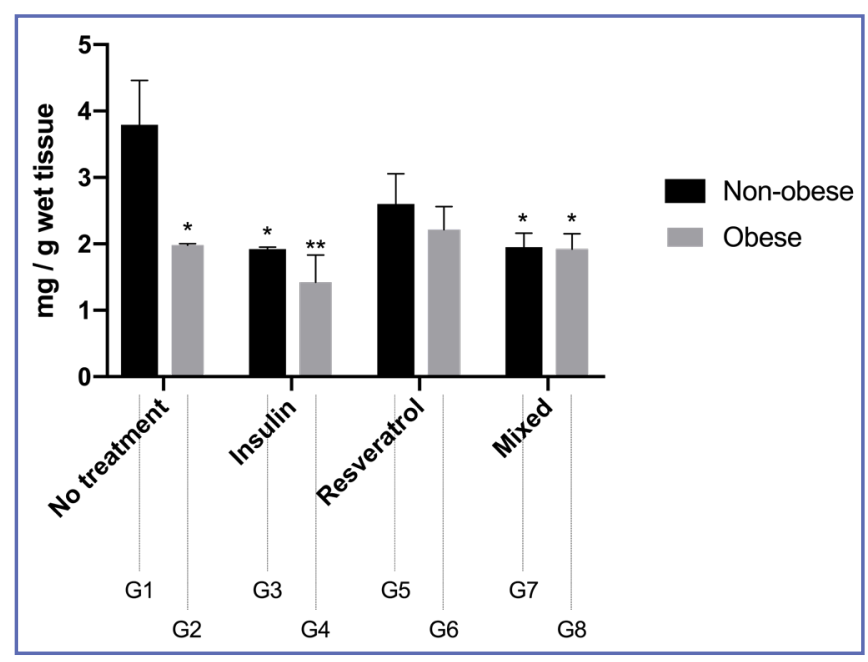

Figure 3. The concentrations of non-collagenous proteins (NCP). Asterisk designates statistical difference from non-obese and untreated animals. $* \mathrm{p}<0.05 ; * * \mathrm{p}<0.01$.
It was observed that the obese untreated animals present higher quantities of MMPs since obesity establishes a chronic tendon lesion (2) which will need intense matrix degradation to repair. The obese group treated with resveratrol had a lower amount of active MMP-2 compared to the obese group treated with insulin. Samples with lower MMPs concentration are probably undergoing less degradative and inflammatory processes, revealing RSV anti-inflammatory efficiency (10).

Injured tendons present reduction in the concentration of NCPs (11). Obesity alone caused a significant decrease, which was also detected in non-obese animals that received insulin (associated or not with RSV). Alternatively, the mice treated only with RSV (obese or otherwise) did not present any reduction in the concentration of NCP. When comparing non-obese animals with the use of insulin and without, it is possible to notice that insulin alone, just like obesity, cause a reduction in the concentration of NPC in the tendon. RSV

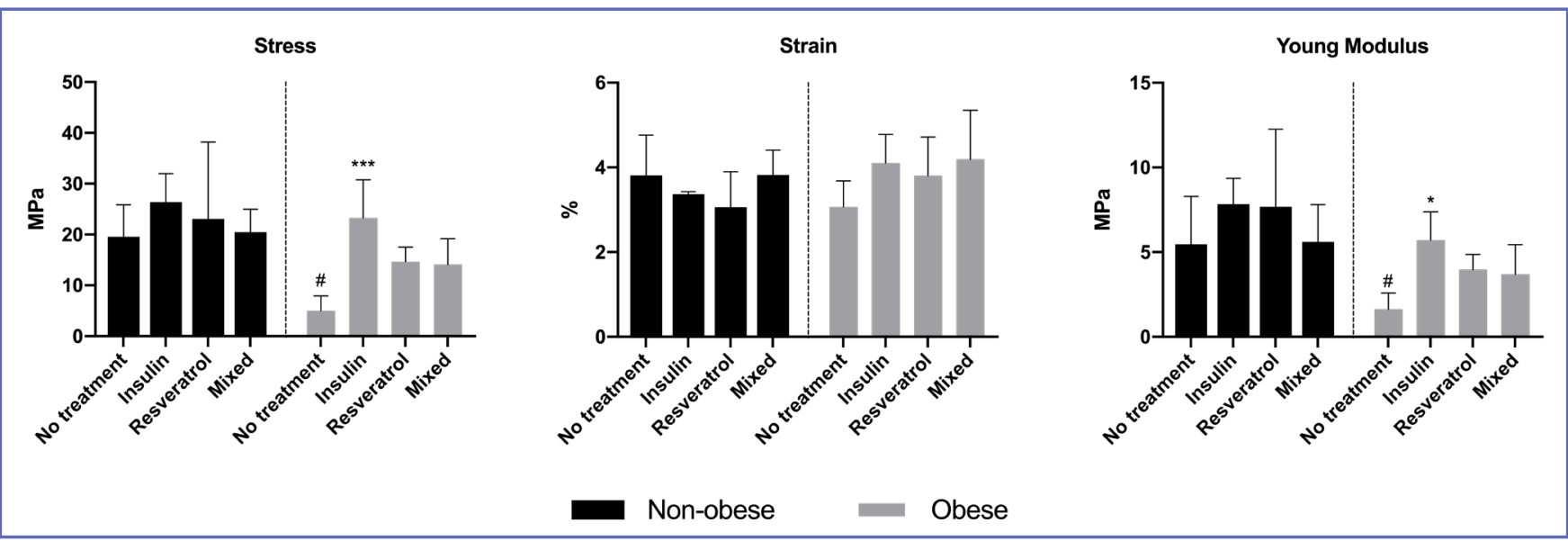

Figure 4. Stress and Strain values of the evaluated tendons. No significative difference could be observed in the strain of the different groups. Obesity alone decreased the tendon strength when compared to all non-obese animals (\#). Insulin treatment made the tendon as resistant as the non-obese animals' tendons.

The * mean the value is different from obese untreated animals. *p $<0.05 ; * * \mathrm{p}<0.01 ; * * * \mathrm{p}<0.001 ; * * * * \mathrm{p}<0.0001$.

\section{DISCUSSION}

In this study, the connection between the obese insulin-resistant mouse and changes in calcaneal tendon was explored. Obese, with or without treatment, have alterations in MMP-2 and MMP-9 isoforms activity which can cause modification in molecular levels in tendons. Interestingly, the activity of MMP-9 increases in the tendons of obese mice in comparison with lean mice, while treatment with resveratrol was able to decrease it. It was noticed that RSV acts reducing the activity of important MMPs in the tendons of insulin-resistant obese animals. on the other hand demonstrated a protective effect, preserving the content of this component.

IR may cause complications in tissues rich in collagen due to non-enzymatic glycosylation - reduction of sugars in the proteins - which generates structural changes in collagen, resulting in biomechanical damage (7). In the biomechanical tests performed, the parameters analyzed were: stress, which corresponds to the strength needed to cause the tendon rupture; strain, refers to how much that the tendon could be stretched before the rupture; finally, The Young Modulus, analyzes whether the tendon is elastic or if its structure is rigid. A high- 
er Stress means the tension resistance is better preserved due to the insulin treatment of obese animals (19). When it comes to strain, no significant difference was observed between the groups. The results observed from The Young Modulus shows that insulin treatment is more efficient to maintain the tendon's elasticity. Obesity, as expected, was able to decrease the tendon strength, and Young Modulus; which is also related to the fact that IR patients are more susceptible to tendon lesions and have their biomechanical properties compromised (19). Other studies also demonstrated that tendons of IR animals are thickened, presents decreased Young's modulus and tensile stress, in addition to a reduced resistance to maximum load with a lower peak failure force $(6,11)$.

Biomechanical results demonstrated that insulin treatment improved tendon properties, while resveratrol did not bring significant changes. The obese animals receiving the combined treatment showed no improvement compared to the control. These data suggest a possible interaction between insulin and RSV that led to the reduction of insulin positive effects. It is possible that RSV caused an insulin reduction, thus reverting its positive effects over tendon biomechanics (20). According to Zhu, even though RSV regular consumption improves glucose homeostasis and reverses insulin resistance, the results available in the literature are inconsistent, possibly due to a lack of standardization in the doses used (21).

Due to its biological properties, RSV plays a fundamental role in tendon healing on IR and DM2 patients. The anti-diabetic effects can increase the activity and number of GLUT - involved in glucose transportation, protects pancreatic cells from oxidative stress and reverses insulin resistance by reducing body fat mass (3). Also, RSV enhanced the production of matrix components, such as collagen types I and III, whereas it inhibited gene products involved in inflammation and apoptosis (22). In this context RSV effects over inflammation and diabetes could be helpful addressing DM2 patients, helping not only to control the disease but also to make the tendon lesion treatment more efficient.

According to Ackerman et al. (2017) (2) a diabetic tendon will have an increase in thickness and number of ECM cell numbers, since DM2 alters tendon homeostasis and impairs the healing process. In an attempt of recovering, there is an overexpression of MMPs genes. During repair three phases can be observed: inflammatory, proliferative, and remodeling phase. During the inflammatory phase, there is intense

\section{REFERENCES}

1. Nandi A, Kitamura Y, Kahn CR, Accili D. Mouse models of Insulin Resistance. Physiol Rev 2004;84(2):623-47.
ECM degradation, explaining the presence of MMP-2 and -9. Throughout the remodeling phase, collagen III (synthesized during proliferative phase) is replaced by collagen I, with the presence of MMP-2 (23).

A study using human tenocyte culture model, with IR induced by TNF, demonstrated the process of tenocytes homeostasis in diabetic individuals with tendinopathy. It was observed that tenocytes from injured tendons produce a greater amount of type III collagen, when compared to an intact tendon. In addition, tendon homeostasis is modulated by MMPs and TIMPs (tissue inhibitors of MMPs), which regulates the production of collagen and non-collagen matrix by tenocytes. Thus, it was shown that IR positively regulates the expression of MMP-9, highlighted here, and MMP-13 (24). Another study demonstrated that RSV, as an antioxidant, can decrease MMP-9 expression not only by suppressing MMP-9 production but also by augmenting tissue inhibitors of metalloproteinases (TIMPs) production (25).

The mechanism through which RSV acts over obese tendons is still unclear and needs further clarification. Also, future studies evaluating different RSV doses over animal treatment are necessary.

\section{CONCLUSIONS}

Resveratrol treatment demonstrated a protective effect over tendons in obese insulin-resistant animals, reducing the activity of different MMPs, MMP-2, and MMP-9, and avoiding protein content reduction. In addition to that, it was noticed that tendons from obese mice had alterations in the profile of the tested MMPs, and strain and elasticity compromised. Overall, this study showed that the response of MMP-2 and MMP-9 are high in obese, yet RSV treatment was able to reduce it. Nonetheless, insulin and RSV combination reduced insulin efficiency over the biomechanical properties tested.

In order to propose an alternative treatment that could benefit the patients, more studies are necessary to elucidate how RSVs antidiabetic properties act. It's also important to determine efficient doses and the best means of administration.

\section{CONFLICT OF INTERESTS}

The authors declare that they have no conflict of interests. 
resulting in a fibrotic tendon healing response. PLoS One 2017;12(7):e0181127.

3. Rehling T, Bjørkman A-SD, Andersen MB, Ekholm O, Molsted S. Diabetes is associated with musculoskeletal pain, osteoarthritis, osteoporosis, and rheumatoid arthritis. J Diabetes Res 2019;2019:1-6.

4. Bedi A, Fox AJS, Harris PE, et al. Diabetes mellitus impairs tendon-bone healing after rotator cuff repair. J Shoulder Elbow Surg 2010;19(7):978-88.

5. Volper BD, Huynh RT, Arthur KA, et al. Influence of acute and chronic streptozotocin-induced diabetes on the rat tendon extracellular matrix and mechanical properties. Am J Physiol Regul Integr Comp Physiol 2015;309(9):R1135-43.

6. Oliva F, Piccirilli E, Berardi AC, Frizziero A, Tarantino U, Maffulli N. Hormones and tendinopathies: the current evidence. Br Med Bull 2016;117(1):39-58.

7. Bezerra MA, da Silva Nery C, de Castro Silveira PV, et al. Previous physical exercise slows down the complications from experimental diabetes in the calcaneal tendon. Muscles Ligaments Tendons J 2016;6(1):97-103.

8. Guerra FDR, Vieira CP, dos Santos de Almeida M, et al. Pulsed LLLT improves tendon healing in rats: a biochemical, organizational, and functional evaluation. Lasers Med Sci 2014;29(2):805-11.

9. Rehman K, Saeed K, Munawar SM, Akash MSH. Resveratrol regulates hyperglycemia-induced modulations in experimental diabetic animal model. Biomed Pharmacother 2018;102:140-6.

10. Zeytin K. The effects of resveratrol on tendon healing of diabetic rats. Acta Orthop Traumatol Turc 2014;48(3):355-62.

11. Boivin GP, Elenes EY, Schultze AK, Chodavarapu H, Hunter SA, Elased KM. Biomechanical properties and histology of $\mathrm{db} / \mathrm{db}$ diabetic mouse Achilles tendon. Muscles Ligaments Tendons J 2014;4(3):280-4.

12. Silva MBG, Skare TL. Manifestações musculoesqueléticas em diabetes mellitus. Rev Bras Reumatol 2012;52(4):601-9.

13. Padulo J, Oliva F, Frizziero A, Maffulli N. Muscles Ligaments and Tendons Journal - Basic principles and recommendations in clinical and field Science Research: 2018 update. Muscles Ligaments Tendons J 2018;8(3):305-7.

14. André DM, Calixto MC, Sollon C, et al. Therapy with resveratrol attenuates obesity-associated allergic airway inflammation in mice. Int Immunopharmacol 2016;38:298-305.
15. Rieder SA, Nagarkatti P, Nagarkatti M. Multiple anti-inflammatory pathways triggered by resveratrol lead to amelioration of staphylococcal enterotoxin B-induced lung injury: Therapeutic role of resveratrol in acute lung injury. Br $\mathrm{J}$ Pharmacol 2012;167(6):1244-58.

16. Calixto MC, Lintomen L, André DM, et al. Metformin attenuates the exacerbation of the allergic eosinophilic inflammation in high fat-diet-induced obesity in mice. PLoS One 2013;8(10):e76786.

17. Marqueti RC, Parizotto NA, Chriguer RS, Perez SEA, Selistre-de-Araujo HS. Androgenic-anabolic steroids associated with mechanical loading inhibit matrix metallopeptidase activity and affect the remodeling of the Achilles tendon in rats. Am J Sports Med 2006;34(8):1274-80.

18. Bradford MM. A rapid and sensitive method for the quantitation of microgram quantities of protein utilizing the principle of protein-dye binding. Anal Biochem 1976;72:248-54.

19. Wu Y-F, Huang Y-T, Wang H-K, Yao C-CJ, Sun J-S, Chao Y-H. Hyperglycemia augments the adipogenic transdifferentiation potential of tenocytes and is alleviated by cyclic mechanical stretch. Int J Mol Sci 2017;19(1):90.

20. Jimoh A, Tanko Y, Ayo JO, Ahmed A, Mohammed A. Resveratrol increases serum adiponectin level and decreases leptin and insulin level in an experimental model of hypercholesterolemia. Pathophysiology 2018;25(4):411-7.

21. Zhu X, Wu C, Qiu S, Yuan X, Li L. Effects of resveratrol on glucose control and insulin sensitivity in subjects with type 2 diabetes: systematic review and meta-analysis. Nutr Metab (Lond) 2017;14:60.

22. Busch F, Mobasheri A, Shayan P, Lueders C, Stahlmann R, ShakibaeiM. Resveratrol modulates interleukin-1 $\beta$-induced phosphatidylinositol 3-kinase and nuclear factor $\kappa B$ signaling pathways in human tenocytes. J Biol Chem 2012;287(45):38050-63.

23. Campos ACL, Borges-Branco A, Groth AK. Cicatrização de feridas. Arq Bras Cir Dig 2007;20(1):51-8.

24. Tan HY, Tan SL, Teo SH, Roebuck MM, Frostick SP, Kamarul T. Development of a novel in vitro insulin resistance model in primary human tenocytes for diabetic tendinopathy research. PeerJ 2020;8:e8740.

25. Farrokhi E, Ghatreh-Samani K, Salehi-Vanani N, Mahmoodi A. The effect of resveratrol on expression of matrix metalloproteinase 9 and its tissue inhibitors in vascular smooth muscle cells. ARYA Atheroscler 2018;14(4):157-62. 